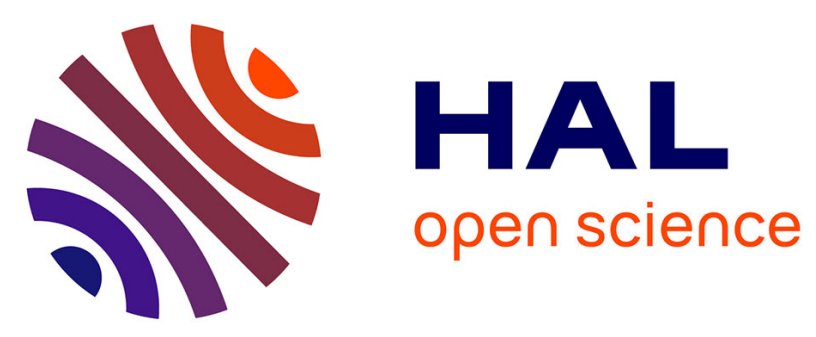

\title{
A Workflow for Multiclass Determination of 256 Pesticides in Essential Oils by Liquid Chromatography Tandem Mass Spectrometry using Evaporation and Dilution Approaches: Application to Lavandin, Lemon and Cypress Essential Oils
}

Yoann Fillâtre, David Rondeau, Antoine Daguin, Pierre-Yves Communal

\section{To cite this version:}

Yoann Fillâtre, David Rondeau, Antoine Daguin, Pierre-Yves Communal. A Workflow for Multiclass Determination of 256 Pesticides in Essential Oils by Liquid Chromatography Tandem Mass Spectrometry using Evaporation and Dilution Approaches: Application to Lavandin, Lemon and Cypress Essential Oils. Talanta, 2016, 149, pp.178-186. 10.1016/j.talanta.2015.11.052 . hal-01240770

HAL Id: hal-01240770

https://hal-univ-rennes1.archives-ouvertes.fr/hal-01240770

Submitted on 7 Jan 2016

HAL is a multi-disciplinary open access archive for the deposit and dissemination of scientific research documents, whether they are published or not. The documents may come from teaching and research institutions in France or abroad, or from public or private research centers.
L'archive ouverte pluridisciplinaire HAL, est destinée au dépôt et à la diffusion de documents scientifiques de niveau recherche, publiés ou non, émanant des établissements d'enseignement et de recherche français ou étrangers, des laboratoires publics ou privés. 
A Workflow for Multiclass Determination of 256 Pesticides in Essential Oils by Liquid Chromatography Tandem Mass Spectrometry using Evaporation and Dilution Approaches: Application to Lavandin, Lemon and Cypress Essential Oils.

Yoann Fillatre, ${ }^{\text {a, b,i,* }}$ David Rondeau, ${ }^{\mathrm{c}, \mathrm{d}}$ Antoine Daguin ${ }^{\mathrm{a}}$ and Pierre-Yves Communal ${ }^{\mathrm{a}, \mathrm{e}}$

${ }^{\text {a }}$ Groupement Interrégional de Recherche sur les Produits Agropharmaceutiques (GIRPA), 9 avenue du Bois de l'Abbé, Angers Technopole, 49071 Beaucouze, France.

${ }^{\mathrm{b}}$ Institut des Sciences et Technologies Moléculaires d'Angers (MOLTECH), CNRS UMR 6200, Université d'Angers, 2 boulevard Lavoisier, 49045 Angers Cedex 01, France.

${ }^{\mathrm{c}}$ Institut d'Electronique et de Télécommunication de Rennes, CNRS UMR 6164, Université de Rennes 1, Campus de Beaulieu, 263 Avenue du General Leclerc, 35042 Rennes Cedex, France.

d Université de Bretagne Occidentale, Département de Chimie, 6 Avenue le Gorgeu, 29238 Brest Cedex 03, France.

${ }^{\mathrm{e}}$ Laboratoire d'Etudes Environnementales des Systèmes Anthropisés (LEESA), Université d'Angers, 2 boulevard Lavoisier, 49045 Angers Cedex 01, France.

*Corresponding author

Yoann Fillatre: Phone: +33(0) 489856014. E-mail: yoann.fillatre@erini-institute.com.

\section{Abstract}

This paper describes the determination of 256 multiclass pesticides in cypress and lemon essential oils (EOs) by the way of liquid chromatography-electrospray ionization tandem mass spectrometry (LC-ESI/MS/MS) analysis using the scheduled selected reaction monitoring mode (SSRM) available on a hybrid quadrupole linear ion trap (QLIT) mass spectrometer. The performance of a sample preparation of lemon and cypress EOs based on dilution or evaporation under nitrogen assisted by a controlled heating were assessed. The best limits of quantification (LOQs) were achieved with the evaporation under nitrogen method giving LOQs $\leq 10 \mu \mathrm{g} \mathrm{L}^{-1}$ for $91 \%$ of the pesticides. In addition the very satisfactory results obtained for recovery, repeatability and linearity showed that for EOs of relatively low evaporation temperature, a sample preparation based on evaporation under nitrogen is well adapted and preferable to dilution. By compiling these results with those previously published by some of us on lavandin EO, we proposed a workflow dedicated to multiresidue determination of pesticides in various EOs by LC-ESI/sSRM. Among the steps involved in this worflow, the protocol related to mass spectrometry propose an alternative confirmation method to the classical SRM ratio criteria based on a SSRM survey scan followed by an information-dependent acquisition using the sensitive enhanced product ion (EPI) scan to generate MS/MS spectra then compared to a reference. The submitted workflow was applied to the case of lemon EOs samples highlighting for the first time the simultaneous detection of 20 multiclass pesticides in one EO. Some pesticides showed very high concentration levels with amounts greatly exceeding the $\mathrm{mg} \mathrm{L}^{-1}$.

Keywords

Pesticides, lemon, cypress, essential oil, LC-MS/MS, sample preparation.

\section{Introduction}

The market of EOs is rapidly expanding and generates several billions of dollars every year.[1] The importance and the development of the EOs' market can be explained by the number and diversity of application areas of these natural extracts. Most of these application fields are directly related to human consumption such as cosmetics and perfumes,[2] medicine,[3,4] food,[4-7] agriculture,[8-11] as well as aromatherapy.[1,4,12] The cultivation of EOs' raw material (medicinal and aromatic plants) commonly involves the use of pesticides, which can concentrate through the extraction process. [13] This is evidenced by the fact that pesticide residues have been already found in EOs of citrus,[14-17] boronia,[18] fennel [18] or parsley.[18] However, unlike foodstuffs as water or fruits and vegetables for which maximum residues limits (MRLs) are established and available for consultation in databases (Europe: http://ec.europa.eu/sanco_pesticides/public/index.cfm; Worldwide: $\mathrm{http}: / / w w w . m r l d a t a b a s e . c o m)$, there is no regulation in regards to pesticides contents in EOs.[19] The presence of pesticide residues in EOs can lead to a public health problem, particularly due to the rapid expansion of aromatherapy market and its by-products. Furthermore, this practice also tends to individualize through the

\footnotetext{
${ }^{\mathrm{i}}$ Present address: European Research Institute on Natural Ingredients (ERINI), Espace Jacques-Louis Lions - 4 Traverse Dupont, 06130 Grasse, France.
} 
publication of guides or manuals of EOs referencing all the virtues of these extracts, as well as various methods of preparation for practice at home.[20] This is possible thanks to the free access and use of EOs to the whole people. In this context, laboratories have to dispose of multiresidue analytical methods to control pesticides in EOs to assure consumer's health, waiting for regulation limits.

The possibility to determine several hundred of pesticides in drinking or rinsing water by LC-MS/MS has been often reported in literature.[21-24] In this context some of us have shown the advantages in terms of data points per peaks, reproducibility, signal-to-noise ratio and number of SRM transitions, of using the sSRM algorithm available on a hybrid quadrupole linear ion trap (QLIT) mass spectrometer by comparison to the classical SRM mode.[25] For more complex matrixes such as EOs, the challenge is not only related to the number of residue to be detected and quantified but also to the sample preparation to consider for obtaining the best results in terms of limit of detection (LOD) and quantification (LOQ) and recovery rate. In fact, as highlighted in a recent review,[19] relatively few papers are dedicated to pesticides analysis in EOs and the available methods are quite limited in terms of LODs and number of simultaneously analyzed pesticides.[13,14,16,26-29]

The classical approaches for the multiresidue analyses of pesticides in foodstuffs mention the use of sample preparations methods such as the Solid-Phase Extraction (SPE),[30-33] the Pressurized Liquid Extraction (PLE)[34-36] or the recent normalized QuEChERS (Quick, Easy, Cheap, Effective, Rugged and Safe) method that could be hardly applicable to Eos.[37-42] Our aim was rather to consider the simplest possible sample preparation method. In the case of the multiresidue analysis of 70 multiclass pesticides in lavandin EO, a sample pre-treatment consisting of a 10 -fold dilution in methanol $(\mathrm{MeOH})$ acidified with $1 \%$ of acetic acid has been considered before the $20 \mu 1$ sample injection in the LC-MS/MS device operating in sSRM acquisition mode.[43] Interesting results were obtained since 62 of the 70 pesticides gave LOQs below $10 \mu \mathrm{g} \mathrm{L}^{-1}$.[43] The feasibility of applying this dilution method to a higher number of pesticides, i.e. 256 active substances in lavandin EO was tested.[44] In order to improve the LOQs, an enrichment step was envisaged. Based on evaporation under nitrogen assisted by a controlled heating, this alternative preparation method was tested and allowed an improvement of 92 pesticide's LOQ. This study showed however that the evaporation method was not suitable for the validation of a quantification method for every pesticide because the high temperature required to evaporate lavandin EO led to the loss of some of them by thermal degradation. [44] However, the evaporation under nitrogen could be considered as a complementary method to the sample preparation by dilution in the multiresidue determination of pesticides in lavandin EO. Nevertheless, the use of the evaporation under nitrogen for the determination of EOs characterized by a lower evaporation temperature should be more efficient and could be considered as a method of choice to assure optimal detection of all active substances in such matrixes. In this context, it can be interesting to elaborate a workflow for the analysis of pesticides in EOs that should take in account two different protocols depending on the evaporation temperature of the sample matrix. Furthermore, beyond the selection of SRM transitions usually considered to detect and quantify the presence of pesticides, the analytical method can be improved by taking into account of additional fragmentations that can be used as confirmation to avoid the production of false positives and negatives.[41,45,46] In this goal, we have used the advantages of the hybrid triple quadrupole linear ion trap (LIT) technology (QTRAP ${ }^{\circledR}$ ) that are namely related to the possibility of simultaneous detection of a pesticide and the confirmation of its identification by informationdependent acquisition (IDA) considering a SRM acquisition mode as the survey scan and an enhanced product ion (EPI) mode as the dependent scan.[47-49]

In this paper we present first the comparison between the performance of dilution and evaporation under nitrogen methodologies in case of pesticides determination in lemon and cypress EOs that are characterized by their low flash point, $46{ }^{\circ} \mathrm{C}[50]$ and $35^{\circ} \mathrm{C},[51]$ respectively. This important step in the elaboration of our worflow shows the advantages of the sample preparation by evaporation for these EOs. Then, thanks to the results previously obtained with the lavandin EO, a workflow for the pesticides residue determination in EOs is proposed, namely because the three EOs analysed are characterized by their difference in chemical composition and the fact that they are extracted respectively either by expression [50] and steam distillation. [51,52] Finally, this procedure was applied to real lemon EO samples.

\section{Materials and Methods}

\section{Pesticides and Reagents}

HPLC-grade MeOH and acetic acid were supplied by SDS (Peypin, France). Ammonium acetate (99.1\% purity) was purchased from VWR (Fontenay-sous-bois, France). Water used for LC-MS/MS analysis was generated from a SERALPUR Pro $90 \mathrm{CN}$ system (SERAL, France). Pesticide standards of analytical grade were purchased from Cil Cluzeau (Sainte-Foy-La-Grande, France) or from Sigma-Aldrich (Saint-Quentin Fallavier, France). Individual standard stock solutions were prepared by dissolving the standard in acetonitrile (ACN) or $\mathrm{MeOH}$ to reach the final concentration of $1 \mathrm{~g} \mathrm{~L}^{-1}$. Ten standard mixtures were prepared from individual stock solutions and diluted with $\mathrm{ACN}$ to yield a concentration of $10 \mathrm{mg} \mathrm{L}^{-1}$. Parent compounds and metabolites were separated in 
different mixtures to check for possible decomposition. These ten solutions were then mixed to obtain a final standard mix solution in ACN at $1 \mathrm{mg} \mathrm{L}^{-1}$, used to fortify samples. Note that benomyl and carbendazim were not included in the mixture in order to study the degradation of thiophanate-methyl during the evaporation process.

\section{Sample preparation}

Two preparation methods were assessed: the "dilution method" and the "evaporation under nitrogen method". The first one, as described elsewhere,[43] consisted of a 10-fold dilution in $\mathrm{MeOH}$ acidified with $1 \%$ of acetic acid. For the evaporation method, $500 \mu \mathrm{L}$ of lemon and cypress EOs were evaporated to dryness under a gentle nitrogen flow and assisted by heating with a temperature of $45^{\circ} \mathrm{C}$. The time required for this step was about 40 minutes. The residues were then dissolved in $500 \mu \mathrm{L}$ of MeOH containing $1 \%$ of acetic acid to reach a 1:1 extraction ratio.

Starting from the final standard mix solution at $1 \mathrm{mg} \mathrm{L}^{-1}$, organic lemon and cypress EOs were spiked at five concentrations: $1,5,10,25$ and $50 \mu \mathrm{g} \mathrm{L}^{-1}$. These samples then followed the dilution and evaporation under nitrogen protocols and were used to build calibration curves. In addition, to assess the recovery and repeatability of the evaporation under nitrogen method for all pesticides, quintuplicate were done for 10 and $50 \mu \mathrm{g} . \mathrm{L}^{-1}$ concentration levels. The recoveries were evaluated based on two matrix-matched calibration points (10 and 50 $\mu \mathrm{g} \mathrm{L}^{-1}$ ) prepared with $90 \%$ of blanks EOs and $10 \%$ of standards in $\mathrm{MeOH}$.

The presence of waxes in lemon EO leads to solubility issues with $\mathrm{MeOH}$. It is even more pronounced when the evaporation under nitrogen is used due to the concentration of the matrix component (extraction ratio 1:1). To overcome this problem, a common way in pesticides residue analysis is to perform a freeze out step. [53-56] The developed protocol consists in a precipitation of the diluted extract in liquid nitrogen (4 minutes) followed by a cold centrifugation ( 5 minutes) and a direct sampling of the supernatant. Note that an overnight freezing $(<15$ ${ }^{\circ} \mathrm{C}$ ) was also tested and gave similar results in terms of pesticides' recovery. (Unpublished data) However the freeze out based on liquid nitrogen was faster and seems to remove more waxes from a visual aspect.

\section{Liquid Chromatography}

LC was performed with an Ultimate 3000 (Dionex, Voisins Le Bretonneux, France) HPLC system equipped with a binary pump. Chromatographic separation was achieved on a Synergy Hydro-RP C18 LC column (150 mm $\times$ $4.6 \mathrm{~mm}$ i.d., $4 \mathrm{~mm}$ particle size; Phenomenex, Le Pecq, France). The oven temperature was kept at $35{ }^{\circ} \mathrm{C}$ and the temperature of the autosampler was set at $4{ }^{\circ} \mathrm{C}$ to limit the decomposition of thermolabile pesticides.

Compounds were separated with a gradient of $\mathrm{MeOH}$ and water. Eluent A consisted of water, $0.1 \%$ of acetic acid and $5 \mathrm{mM}$ of ammonium acetate, and eluent $\mathrm{B}$ consisted of $\mathrm{MeOH}$ with the same amount of acetic acid and ammonium acetate. The mobile phase flow rate was maintained at $1 \mathrm{~mL} / \mathrm{min}$ and the gradient was as follows: $\mathrm{t}=$ $0,50 \% \mathrm{~B} ; \mathrm{t}=1 \min , 50 \% \mathrm{~B} ; \mathrm{t}=5 \min , 70 \% \mathrm{~B} ; \mathrm{t}=9 \min , 70 \% \mathrm{~B} ; \mathrm{t}=16 \min , 100 \% \mathrm{~B} ; \mathrm{t}=20 \min , 100 \% \mathrm{~B}, \mathrm{t}$ $=22 \mathrm{~min}, 50 \% \mathrm{~B}$, until $27 \mathrm{~min}$. The injection volume was $20 \mu \mathrm{L}$ for all the extracts.

\section{Targeted Screening Method in Mass Spectrometry}

The LC system was coupled to a 4000 QTRAP ${ }^{\circledR}$ mass spectrometer (AB Sciex, Courtaboeuf, France) equipped with a turbo ion spray interface (electrospray ionization) operated both in positive and negative ionization mode. The source conditions were the following: ionspray voltage $5500 \mathrm{~V}$ in positive mode and $-4500 \mathrm{~V}$ in negative mode; curtain gas pressure, 35 psi; nebulizer gas and heating gas (GS1 and GS2) pressure, 40 and 50 psi, respectively. The source temperature was set at $550{ }^{\circ} \mathrm{C}$. Compressed air was used as the nebulizer and heating gas. Nitrogen was used as the curtain and collision gas. The values of the declustering potential (DP), the collision energy (CE) and the cell exit potential (CEP) were optimized for each analyte (see Table S1 in Supplementary material section). The collision gas pressure indication was set to "high" in order to maximize the fragmentation. Data acquisition was performed with the sSRM mode with a retention time window of $60 \mathrm{~s}$ and a target scan time of $1.3 \mathrm{~s}$ (isomers requiring separated detection windows were considered independently). Two SRMs transition were acquired for the 23 pesticides analyzed in ESI- mode allowing a confirmation of the residue by use of the SRM ratio criteria. ${ }^{53}$ Regarding the analysis in ESI+ mode, one SRM transition was acquired for the 233 pesticides to optimize the sensitivity of each analyte.[25] The confirmation of the residue was done using a second analysis based on EPI using IDA.

\section{Confirmatory Method in Mass Spectrometry}

The survey scan was composed of the SRM transitions only related to the pesticides suspected to be positive during the screening analysis. IDA criteria were composed of an include list of the ions and retention time (RT) of the positive pesticides and an intensity threshold of 100 counts. When a SRM transition matched the IDA 
criteria, the mass spectrometer switched in EPI mode to generate the mass spectrum of the pesticide. Parameters for EPI mode were the following: DP $40 \mathrm{~V}$; fill time: $80 \mathrm{~ms}$; CE/CES (Collision Energy Spread): 40+/-20 V; LIT acquisition speed: $1000 \mathrm{Da} / \mathrm{s}$; Q1 resolution: High.

\section{Essential Oils}

Lemon and cypress EOs from organic production, i.e. by considering any mentioned phytosanitary treatment, were obtained from a private company. Note that before considering these two matrixes spiked with the pesticides to be determined, the LC-MS-MS targeted screening method described above, was applied to the lemon and cypress EOs. By referring to the retention times and SRM transitions reported in Table S1, significant responses corresponding to different residues were highlighted. Indeed, 16 pesticides appears to be detectable in the lemon EO, i.e. azaconazole, bitertanol, chlorpyrifos, fenamiphos, fenpropimorph, fenthion sulfoxide, hexazinone, hexythiazox, malathion, mefenoxam, mepronil, methidathion, metribuzin, oxamyl, phosmet and propyzamide versus 7 in the cypress EO, i.e. benalaxyl, cyprodinil, dimethachlor, metolachlor, prosulfocarb and tebufenpyrad. For most cases, blank values were not higher than $30 \%$ of the reporting LOQ, so recovery were calculated using blank subtracted calibration.[57] However in the case of azaconazole, metribuzin and phosmet the signals were too intense and no results could be reported.

\section{RESULTS AND DISCUSSION}

\section{Advantage of a Sampling Method by Evaporation for EOs with Relatively Low Evaporation Temperature}

The performance of the evaporation under nitrogen method can be described through the Recovery Rate (R) and the Relative Standard Deviation (RSD) of pesticides determined from the lemon and cypress EOs. The Table S2 regroups the means recovery and the RSDs obtained from the analysis of 256 pesticides spiked at concentrations of 10 and $50 \mu \mathrm{g} \mathrm{L}^{-1}(\mathrm{n}=5)$ in the organic lemon and cypress EOs, performed from a sampling using the evaporation under nitrogen method.

For a better reading of these results, the data obtained after the $50 \mu \mathrm{g} \mathrm{L}^{-1}$ mixture spiking are synthesized by the bar charts of Fig. 1 which depicts the percentage of active substances respectively function of mean recovery (Fig. 1a) and RSD (Fig. 1b) for each pesticide and for the two EOs.

Fig. 1. Percentage of pesticides function of a) the mean recovery (\%) and b) the RSD (\%) during the evaporation step for the $50 \mu \mathrm{g} \mathrm{L}^{-1}$ spiking concentration $(\mathrm{n}=5)$.

From Fig. 1 and Table S2, it appears that only two active substances are not detected in lemon EO, i.e. the thiophanate-methyl and the tau-fluvalinate, and also two still undetected in cypress EO, i.e. the resmethrin and the spinosad A. Recoveries of all others pesticides are very satisfactory. Thus, $97.6 \%$ and $94.8 \%$ of active substances had recoveries between $70 \%$ and $120 \%$ in lemon and cypress EOs, respectively. Actually 5 pesticides lemon $E O$, i.e. carboxine $(R=63.0 \%)$, cycloxidim $(R=64.8 \%)$, cyromazine $(R=65 \%)$, dichlorvos $(\mathrm{R}=59.8 \%)$, thiophanate methyl $(\mathrm{R}=0 \%)$ and 10 in cypress $\mathrm{EO}$, i.e. bifenazate $(\mathrm{R}=17.4 \%)$, carbofuran $(\mathrm{R}=$ $39.4 \%)$, carboxin $(\mathrm{R}=67.5 \%)$, clofentezine $(13.1 \%)$, cycloxidim $(\mathrm{R}=66.4 \%)$, dichlorvos $(\mathrm{R}=62.7 \%)$, disulfoton $(\mathrm{R}=63.5 \%)$, fluazinam $(\mathrm{R}=60.6 \%)$, quinoclamine $(\mathrm{R}=22.5 \%)$, thiophanate methyl $(\mathrm{R}=7.8 \%)$ showed recovery below $70 \%$, RSDs were also very good since they are below $20 \%$ for almost all the pesticides. The exceptions concern the amitraz with a RSD value of $30.2 \%$ and $26.4 \%$ in lemon and cypress respectively (explained by the acidity of the injection solvent)[44] and the quinoclamine that presents a RSD value of $64.4 \%$ in cypress. Nevertheless, $90.4 \%$ and $91.3 \%$ of active substances had RSD below $5 \%$ in lemon and cypress EOs, respectively.

The linearity of the methods has been checked for each pesticide through calibration curves. Examples are given in Fig. S1 which depicts the calibration curves of a polar pesticide acephate and a non polar pesticide benalaxyl in lemon and cypress EOs by comparing the dilution and evaporation methods. Matrix effects had already been observed and studied in diluted EOs[43] and as it could be expected, matrix effects are stronger when evaporation under nitrogen is used (Fig. S1). The enrichment of matrix component during the evaporation process can have two consequences. Firstly, it can increase the baseline noise as it was observed for simazine and tebufenpyrad in cypress EO (Fig. S2). And secondly, the higher concentration of co-eluting compounds can cause higher ion suppression during the electrospray ionization process resulting in a loss of sensitivity expressed by a lower slope for evaporation matrix-matched calibration curves compared to the dilution ones (Fig. S1). However if the decrease in the signal response due to matrix effects is not as important as the gain obtained thanks to the concentration factor of 10, we could expected a better sensitivity. Considering the matrixes and the sample preparations, it is obvious that matrix effects will be of great importance. A detailed 
study of matrix effects of different EOs as well as various samples of the same EO on pesticides determination would be relevant but was out of the scope of this paper. However, by taking into the matrix-effects, a correct quantification of the residues is ensured. Indeed linearity of matrix-matched calibration curves was satisfactory for the two sample preparations and combined with good R \% and RSD \% in the case of evaporation under nitrogen.

The values of the LOQs of each pesticide have been determined as elsewhere [44] for the two EOs sampled with the dilution and evaporation under nitrogen methods. The LOQs were not statistically determined. They were assessed as the first calibration point that gave a signal-to-noise ratio greater than 10 . For some transitions, a response could be observed in the blank sample. These signals are due to either an interference or the initial presence of the pesticide itself in the EO, so the LOQ was calculated as three times the blank response, as defined in the SANCO guideline.[57] They are all reported in Table S2. The bar charts displayed in Fig. 2 show the percentage of active substances determined for different LOQ ranges in lemon (Fig. 2a) and cypress EOs (Fig. 2b) and according to the two preparation methods, i.e. dilution and evaporation under nitrogen.

Fig. 2. Percentage of pesticides determined in a) lemon EO and b) cypress EO as a function of their LOQ and the sampling method.

The comparison between the results obtained with the two sample preparation for each EO shows that a marked improvement is observed for pesticide's LOQs in the two EOs when evaporation under nitrogen is used. Regarding lemon EO, the Fig. 2a illustrates that $91.7 \%$ of the pesticides (i.e. 235 molecules) gave a LOQ $\leq 10$ $\mu \mathrm{g} \mathrm{L}{ }^{-1}$ with the evaporation under nitrogen method compared to $81.6 \%$ (i.e. 209 molecules) with the dilution method. In addition, about two times more pesticides have a LOQ $\leq 1 \mu \mathrm{g} \mathrm{L}^{-1}$ with the evaporation method (36.3 $\%$, i.e. 96 molecules) than with dilution ( $18 \%$, i.e. 46 molecules). The evaporation process allowed a relevant improvement of 112 LOQs whereas 142 remained unchanged. By contrast, 2 LOQ values increase with respect to the dilution method. This is the case for thiophanate methyl because of its degradation in carbendazim, as previously reported [44]. This is also the case of propargite for which a higher concentration of components interfering with this residue can be proposed to account for the measured relatively higher LOQ. Similar observation can be made for cypress EO for which the bar charts in Fig. $2 b$ show that $91.0 \%$ of pesticides (233 molecules) gave LOQ $\leq 10 \mu \mathrm{g} \mathrm{L}^{-1}$ with the evaporation method compared to $78.6 \%$ (201 molecules) with the dilution method. As in lemon EO, two times more pesticides have a LOQ $\leq 1 \mu \mathrm{g} \mathrm{L}^{-1}$ with evaporation $(30.5 \%$, i.e. 78 molecules) than with dilution ( $14.1 \%$, i.e. 36 molecules). The evaporation process allowed a relevant improvement of 119 LOQs whereas 133 remained unchanged and 4 increase with respect to the dilution method. From the latter, clofentezine and thiophanate methyl were characterized by low recoveries whereas simazine and tebufenpyrad showed a concentration of interferences of cypress EO (see Fig. S2 in Electronic material section). These results show that the evaporation under nitrogen method using namely a $45^{\circ} \mathrm{C}$ temperature gave better LOQs than dilution for both lemon and cypress EOs. Combined with very good pesticides recoveries, RSD and linearity, the evaporation under nitrogen method is more suitable than dilution for the analysis of pesticides in EOs of lemon and cypress. This conclusion is different to that proposed in our precedent paper devoted to the lavandin EO where the evaporation method under a $80{ }^{\circ} \mathrm{C}$ temperature could only be applied as a complementary method to a sampling by dilution.[44]

\section{Worflow for the Pesticide Residues Determination in EOs}

In view of all data collected during this study and the previous one,[44] a worflow schematically represented in Fig. 3 can be proposed for the multiresidue determination of several hundred of pesticides in EOs by LCESI/sSRM.

Fig. 3. Experimental protocol for the analysis of an EO sample

This workflow starts with the choice of the appropriate sample preparation method that depend on the EO flash point and therefore their evaporation temperature. For EOs of relatively low evaporation temperature (i.e. less than $45^{\circ} \mathrm{C}$ ) such as the lemon and cypress EOs, the use of an evaporation under nitrogen assisted by heating appears to be well adapted and preferable to dilution. In this case, recovery and repeatability (RSDs) are very satisfactory. Moreover, compared to the simple dilution, evaporation under nitrogen significantly improves the LOQs for most of the pesticides. By contrast, for EOs characterized by higher evaporation temperature, e.g. higher than $45^{\circ} \mathrm{C}$, as the lavandin $\mathrm{EO}$, the application of evaporation under nitrogen as sample preparation appears less suitable. The warm up of the sample can induce losses of several active substances. In addition, the decrease in recovery rate, sometimes critical $(\mathrm{R}<20 \%)$, and increased RSDs, complicate the quantification of certain pesticides. In this case, even though the dilution method is chosen for the residues determination, the evaporation under nitrogen method can maintain some interests in so far as it leads to an improvement in the 
sensitivity of a considerable number of compounds. It will be only applied to this kind of EO as a complementary method to the dilution.

After one or the other of this so-called sample preparation involving the dilution or evaporation method, the solubilisation in the injection solvent (i.e. MeOH 1\% acidified) has to be checked. In case of solubility issues with the injection solvent as it is expected at least for EOs of citrus, it is advised to perform a freeze-out step in liquid nitrogen as indicated in Fig. 3 to remove waxes. An alternative solution consisting in placing the sample in a freezer $\left(<15^{\circ} \mathrm{C}\right)$ overnight can also be used.

A chromatographic separation using a binary gradient of $\mathrm{MeOH}$ and water coupled with a detection in ESI/MS/MS using the sSRM mode, will ensure the detection and the quantification of more than 250 pesticides in EOs using the positive and negative ion modes.

Based on the different results obtained as function of the chosen sample preparation, the performance of the analytical method still depends on the nature of the EO. Indeed, considering the variety of EOs in terms of chemical compositions, matrix effect are expected to vary from one EO to another and from one pesticide to another as evidenced by the results in lemon and cypress EOs. In such case, the best strength is the compensation of matrix effects which is often achieved by use of matrix-matched calibration curves. However it appears very complicated to find a blank EO for every type of EO. In addition significant variations in chemical compositions can be observed between different samples of a same type of EO, thus matrix effects can also vary. For these reasons, LOQs for each pesticide will have to be validated for each type of EOs and it is advised to use the standard addition method for quantification.

The standard addition procedure consists in adding a known concentration of pesticide around the same order of the pesticide supposed concentration in the sample. Thus, an accurate quantification requires assessing formerly the initial concentration in the sample. For that purpose, a systematic standard addition of a mix of pesticides at 5 $\mu \mathrm{g} \mathrm{L}^{-1}$ (for dilution) or $50 \mu \mathrm{g} \mathrm{L}^{-1}$ (for evaporation) depending on the preparation method is done for every sample. These concentrations are respectively equivalent to the highest LOQ of pesticides in the extracts after a sample preparation by dilution or evaporation under nitrogen.

The standard addition procedure can be seen as time consuming but it also presents several advantages. Firstly it can highlight the presence of interferences which is not rare in the case of pesticide residues determination in EOs despite the specificity of the SRM mode. Secondly it assures that the LOQs are fulfilled and allows a direct quantification of pesticides with concentration in the range of 10 to $50 \mu \mathrm{g} \mathrm{L}{ }^{-1}$ with a standard addition of $50 \mu \mathrm{g} \mathrm{L}$ ${ }^{1}$ (for evaporation). Finally, it allows assessing the concentration of the other pesticides to adjust the standard addition or establish a dilution in case of a too much intense signal.

By focusing on the workflow step involving the LC-MS/MS analysis (Fig. 3), it's interesting to precise that when the ESI source is used in positive ionization mode where a high number of pesticides is targeted (i.e. 233 active substances), only one SRM transition per residue is selected to optimize the sensitivity.[25] However, with this detection scan in SSRM mode, a confirmation scan is performed by using the EPI mode that leads to confirm the identification of the suspected pesticides by acquiring MS/MS spectra. The use of the so-called IDA mode available with QTRAP ${ }^{\circledR}$ mass spectrometer is illustrated in the present paper through the analyses of two lemon EO samples (vida infra). In negative ion mode, the workflow of Fig. 3 shows that a smaller number of pesticides must be detected. For targeting these 23 residues, the LC-ESI/sSRM analysis can be performed through the monitoring of two SRM transitions. This is here the most common method to confirm a pesticide when the analysis is performed in SRM mode.[57] By considering the SRM ratio calculated from calibration standards, the detection of a residue will be confirmed if its SRM ratio corresponds to that of the standard in a \pm $30 \%$ range.[57] One will note that this methodology can be also applied in positive ion mode when a triple quadrupole mass spectrometer is used for the analysis.

\section{Analyses of Lemons EOs Samples}

Two lemon EOs from organic and non organic origins have been bought on the market as anyone could do. They came from Italy and Spain, respectively. The two EOs were prepared according to the workflow described in Fig. 3. In negative ion mode, the analysis of the non organic lemon EO led to the detection and confirmation of endosulfan sulphate and hexaflumuron at concentrations of $7.0 \mu \mathrm{g} \mathrm{L}^{-1}$ and $4.0 \mu \mathrm{g} \mathrm{L}^{-1}$, respectively. None has been detected in the organic EO. In the positive ion mode, 30 and 20 transitions showed significant signals in the non organic and organic samples, respectively. To avoid false positives, these SRMs have been selected to build an LC-ESI/SRM-EPI method in order to perform a second analysis of the samples. By comparison with pesticides reference EPI spectra (see Table S3 for the organic EO and Table S4 for the non organic EO), the LCESI/SRM-EPI allowed to confirm the presence of 18 pesticides in the lemon non organic EO and 2 in the organic one as shown in Table 1.

Table 1 Residues detected by LC-ESI/sSRM in positive mode and confirmed by the use of the EPI mode during the pesticide determination in the non organic and organic lemon EOs. The calculated concentrations in the 
different samples are given in each case in $\mu \mathrm{g} \mathrm{L}^{-1}$ but also in $\mathrm{mg} / \mathrm{kg}$ to allow a comparison with the Lemon EU MRL referring to the lemon fruit and Citrus oil US EPA MRL referring to lemon EO. Based on the Norm ISO $855,[50]$ a density of $0.85 \mathrm{~kg} \mathrm{~L}^{-1}$ has been used to convert the concentrations form $\mu \mathrm{g} \mathrm{L}^{-1}$ to $\mathrm{mg} \mathrm{kg}^{-1}$.

\begin{tabular}{|c|c|c|c|c|}
\hline Pesticides & $\begin{array}{c}\text { Concentration } \\
\mu \mathrm{g} \mathrm{L}^{-1}\end{array}$ & $\begin{array}{l}\text { Concentration } \\
\mathrm{mg} \mathrm{kg}^{-1}\end{array}$ & $\begin{array}{l}\text { Lemon EU MRL } \\
\qquad \mathrm{mg} \mathrm{kg}^{-1}\end{array}$ & $\begin{array}{l}\text { Citrus oil US EPA MRL } \\
\qquad \mathrm{mg} \mathrm{kg}^{-1}\end{array}$ \\
\hline \multicolumn{5}{|l|}{ Non organic lemon $\mathrm{EO}^{\mathrm{a}}$} \\
\hline Azoxystrobin & 25 & 0.03 & 15 & 40 \\
\hline Buprofezin & 1300 & 1.53 & 1 & 80 \\
\hline Carbendazim & Not Calculated & / & 0.7 & I \\
\hline Chlorpyrifos & 8200 & 9.65 & 0.2 & 20 \\
\hline Etoxazole & 60 & 0.07 & 0.1 & \\
\hline Fenazaquin & 57 & 0.07 & 0.5 & 10 \\
\hline Fenpyroxymate & 98 & 0.12 & 0.5 & 10 \\
\hline Hexythiazox & 490 & 0.58 & 1 & 3 \\
\hline Linuron & 3 & 0.004 & 0.05 & ( \\
\hline Mefenoxam & 13 & 0.02 & 0.5 & 7 \\
\hline Methidathion & 144 & 0.17 & 0.02 & 420 \\
\hline Myclobutanil & 792 & 0.93 & 3 & / \\
\hline Pirimiphos methyl & 127 & 0.15 & 1 & I \\
\hline Prochloraz & 11000 & 12.94 & 10 & / \\
\hline Pyrimethanil & 60 & 0.07 & 10 & l \\
\hline Pyriproxyfen & 2300 & 2.71 & 0.6 & 20 \\
\hline Terbutylazine & 256 & 0.30 & 0.1 & l \\
\hline Thiabendazole & 28 & 0.03 & 5 & 15 \\
\hline \multicolumn{5}{|l|}{ Organic lemon $\mathrm{EO}^{\mathrm{b}}$} \\
\hline Diuron & DNQ & & 0.1 & 3 \\
\hline Mefenoxam & DNQ & / & 0.5 & 15 \\
\hline
\end{tabular}

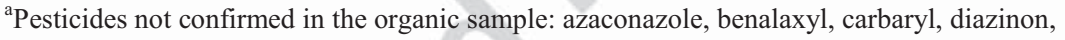
diflufenican, fenamiphos, fenarimol, methamidophos, oxamyl, propargite, sulfotep, trifloxystrobine

${ }^{b}$ Pesticides not confirmed in the non organic sample: azaconazole, buprofezin, carbaryl, chlorpyrifos, cyproconazole, dimethomorph, ethoprofos, fenamiphos, fenarimol, hexythiazox, linuron, malathion, methamidophos, methidathion, oxamyl, propyzamide, rotenone, tetraconazole

From the data of Table 1, it appears that the calculated concentrations in the non organic lemon EO varied between $3 \mu \mathrm{g} \mathrm{L}^{-1}$ for linuron to $11 \mathrm{mg} \mathrm{L}^{-1}$ for prochloraz. It must be mentioned that to our knowledge and until nowadays, citrus EOs were essentially controlled through the determination of organophosphorous and organochlorine pesticides. Among them, chlorpyrifos and methidathion have often been reported to be detected at concentrations above the $\mathrm{mg} \mathrm{L}^{-1}$ in citrus EOs.[17] Then the relatively high concentrations detected in the non organic sample (i.e chlorpyrifos: $8200 \mu \mathrm{g} \mathrm{L}^{-1}$; methidathion : $144 \mu \mathrm{g} \mathrm{L}^{-1}$ ) are not really surprising. However, it should be noted that the use of methidathion as well as endosulfan and hexaflumuron are today formally banned in Europe. In addition these results show for the first time the simultaneous detection and confirmation of twenty chemical active substances belonging to different classes of pesticides in an EO sample.

Besides chlorpyrifos, three other pesticides exceeded a concentration of one $\mathrm{mg} \mathrm{L}^{-1}$. This was the case of buprofezin $\left(1.3 \mathrm{mg} \mathrm{L}^{-1}\right)$, pyriproxyfen $\left(2.3 \mathrm{mg} \mathrm{L}^{-1}\right)$ and prochloraz $\left(11 \mathrm{mg} \mathrm{L}^{-1}\right)$. The only regulatory limits that can be found in Europe are related to the lemon fruit and are detailed in Table 1 for informative purpose. If we dared to compare the concentrations found in the non organic lemon EO with the MRL in the lemon fruit, we would find that seven pesticides (buprofezin, chlorpyrifos, methidathion, pirimiphos-methyl, prochloraz, pyriproxyfen, terbutylazine) exceeded their regulatory limits. Actually, the only MRL that can be found to be specific to citrus EOs have been set by the US EPA for about forty pesticides[59] and are also reported in Table 1 for the concerned residues. Regarding the latter, none exceed these limits. However, it must be recalled here that MRLs are set based on dietary data whereas EOs have many other uses and in particular aromatherapy. 
Finally, one can precise that even if the organic nature of the EO does not insure of the absence of pesticides (diuron and mefenoxam detected), their number and concentrations are much lower $(<\mathrm{LOQ})$ than in the non organic EO.

The interest of an identification scan through the EPI mode in comparison to the use of SRMs ratio criteria is illustrate with the case of the azaconazole. The LC-ESI/sSRM analysis of both lemon EOs showed a significant response for the SRM $1 \mathrm{~m} / \mathrm{z} 300 \rightarrow \mathrm{m} / \mathrm{z} 159$ that is the diagnostic transition of azaconazole (see Fig. 4a and 4b).

Fig. 4. Chromatograms of the analysis of a) a non organic and b) an organic lemon EOs along with c) the reference EPI spectra of azaconazole and the EPI spectra related to the signals observed for the SRM transition $(\mathrm{m} / \mathrm{z} 300 \rightarrow \mathrm{m} / \mathrm{z} 159)$ in the d) non organic and e) organic lemon EOs. The inset shows the results of an additional analysis of the non organic lemon EO with two SRMs (blue $\mathrm{m} / \mathrm{z} 300 \rightarrow \mathrm{m} / \mathrm{z} 159$; red $\mathrm{m} / \mathrm{z} 300 \rightarrow \mathrm{m} / \mathrm{z}$ 231) and showed that a signal is also observed for the second SRM $(\mathrm{m} / \mathrm{z} 300 \rightarrow \mathrm{m} / \mathrm{z} 231)$.

The insert of Fig. 4a displays the results of an additional analysis of the non organic EO which showed that the second SRM of azaconazole (SRM2 m/z $300 \rightarrow \mathrm{m} / \mathrm{z} 231$ ) had also a significant signal that could tend to confirm the presence of azaconazole. In this case, the calculation of the SRM ratio in the EO (SRM2/SRM1 = 44.6) in comparison with the SRM ratio in solvent $(\mathrm{SRM} 2 / \mathrm{SRM} 1=0.45)$ would have clearly led to the non confirmation of its presence. The study of the EPI spectra of azaconazole showed that the product ion spectrum is completely different to the EPI spectrum obtained from the non organic (Fig. 4d) and organic (Fig. 4e) lemon EOs analysis. The fragment ion mass spectrum obtained from collision induced dissociations of the selected $\mathrm{m} / \mathrm{z} 300$ quasimolecular ion of azaconazole, is depicted in Fig. 4c. This MS/MS mass spectrum can be interpreted through the reactivity of the protonated azaconazole molecule under collision activation (see Scheme S1 in Supplementary material section). This is however not the case for the peaks observed in the EPI spectra of Fig. 4d and Fig. 4e that are comparable between them. This suggests that a common interference would be detected in the two EOs. But as the ions m/z 300, m/z 159 and m/z 231 are observed into all depicted mass spectra of the Fig. 4, then such behaviour tends to explain the responses observed with the SRM transitions. In the case of azaconazole, both confirmation techniques (SRM ratio, EPI spectrum) would have led to the report of a false positive. However the use of EPI mode provides more information and seems to be more reliable than SRM ratio since the confirmation is done with the study of the full MS/MS spectra and note only one ion. In addition the use of EPI proved to be very effective with the confirmation of mefenoxam and diuron in the organic lemon EO at very low concentrations, below their LOQ, respectively 5 and $1 \mu \mathrm{g} \mathrm{L}^{-1}$.

In conclusion, through the data collected in the present work and the results previously published, $[25,43,44]$ we propose in this paper a first worflow dedicated to multiresidue determination of several hundred of pesticides in EOs by using a LC-ESI/sSRM approach. This workflow has been built to be applied to EOs of different natures. For the moment, we distinguished two kind of EOs for which the sample preparation will differ: the ones with low evaporation temperature $\left(<45^{\circ} \mathrm{C}\right)$ will be prepared using an evaporation under nitrogen and the others (evaporation temperature $>45^{\circ} \mathrm{C}$ ) will be diluted. Then, in case of solubility issues of the matrix in the injection solvent before the LC-ESI/sSRM analyses, as it is expected at least for the citrus EOs, a freeze out step is added. The LC-ESI/sSRM analysis allowed the detection and quantification of 233 pesticides in ESI+ and 23 pesticides in ESI- at concentration levels below or equal to $10 \mu \mathrm{g} \mathrm{L}^{-1}$ for most of the 256 pesticides. The list of pesticides can be easily increased without observing a significant loss in sensitivity thanks to the use of the sSRM mode.[25] The presence of pesticides can be confirmed either by using the SRM ratio criteria when using a triple quadrupole mass spectrometer or by comparison of the pesticides EPI spectra in the sample with pesticides reference EPI spectra when using a QLIT mass spectrometer. This last alternative allows using only one SRM transition thus optimizing the sensitivity of the detection.

The proposed workflow needs to be enriched with data from others EOs to elaborate a guideline able to cover the wide variety of EOs' chemical compositions. Moreover, even if the matrix effects are compensate with the use of the standard addition methodology, the assessment of matrix effects in each EO would be of great interest with the aim of classing the EOs regarding their matrix effects profile. Therefore, an alternative to the standard addition procedure, which is time-consuming, could be found. This workflow must be also completed with the development of a GC-MS analysis to perform quantification of pesticides not amenable to LC-MS such as the organochlorines. However, the workflow can be applied in its present state as showed by the results obtained from the analyses of two lemon EO samples. It highlighted the presence of respectively 20 and 2 pesticides in the non organic and organic EOs. In the non organic EO, some pesticides such as buprofezin, chlorpyrifos, prochloraz and pyriproxyfen showed high concentration levels with amounts greatly exceeding the $\mathrm{mg} \mathrm{L}^{-1}$. These results show both the effectiveness and the need of the developed methodology. Indeed it allowed for the first time the simultaneous detection and confirmation of twenty residues belonging to different classes of pesticides in a concentration range of $3 \mu \mathrm{g} \mathrm{L}^{-1}$ (linuron) to $11 \mathrm{mg} \mathrm{L}^{-1}$ (prochloraz). The number of detected pesticides and the relatively high amounts for some of them show the importance for laboratories to control pesticide residues in EOs in order to assure consumer's safety. 
References

[1] K.H.C. Baser, G. Buchbauer, Handbook of Essential Oils: Science, Technology, and Applications, Taylor and Francis, 2009.

[2] T. Aburjai, F.M. Natsheh, Plants used in cosmetics, Phytother. Res. 17 (2003) 987-1000. doi:10.1002/ptr.1363.

[3] J. Bruneton, Pharmacognosie: Phytochimie, plantes médicinales, 3e éd. rev. et augm, Tech.\& Doc./Lavoisier, 1999.

[4] A.E. Edris, Pharmaceutical and therapeutic Potentials of essential oils and their individual volatile constituents: a review, Phytother. Res. 21 (2007) 308-323. doi:10.1002/ptr.2072.

[5] S. Burt, Essential oils: their antibacterial properties and potential applications in foods?a review, Int. J. Food Microbiol. 94 (2004) 223-253. doi:10.1016/j.ijfoodmicro.2004.03.022.

[6] B.K. Tiwari, V.P. Valdramidis, C.P. O’Donnell, K. Muthukumarappan, P. Bourke, P.J. Cullen, Application of natural antimicrobials for food preservation, J. Agric. Food Chem. 57 (2009) 5987-6000.

[7] World Health Organization, The world health report 2002 - Reducing Risks, Promoting Healthy Life, WHO, Geneva, 2002.

[8] F.E. Dayan, C.L. Cantrell, S.O. Duke, Natural products in crop protection, Bioorg. Med. Chem. (2009).

[9] R. Garcia, E.S.. Alves, M.P. Santos, G.M.. Aquije, A.A.. Fernandes, R.B. Santos, et al., Antimicrobial activity and potential use of monoterpenes as tropical fruits preservatives, Braz. J. Microbiol. 39 (2008) 163-168.

[10] M.B. Isman, Plant essential oils for pest and disease management, Crop Prot. 19 (2000) 603-608.

[11] M.B. Isman, S. Miresmailli, C. Machial, Commercial opportunities for pesticides based on plant essential oils in agriculture, industry and consumer products, Phytochem. Rev. (2010). doi:10.1007/s11101-010-9170-4.

[12] G. Buchbauer, L. Jirovetz, Aromatherapy — use of fragrances and essential oils as medicaments, Flavour Fragr. J. 9 (1994) 217-222. doi:10.1002/ffj.2730090503.

[13] S.M. Garland, R.C. Menary, N.W. Davies, G.. Oliver, Practical approaches to the analyses for pesticide residues in essential oils, RIRDC, 2004.

[14] G. Di Bella, M. Saitta, L. La Pera, M. Alfa, G. Dugo, Pesticide and plasticizer residues in bergamot essential oils from Calabria (Italy), Chemosphere. 56 (2004) 777-782.

[15] G. Di Bella, L. Serrao, F. Salvo, V.L. Turco, M. Croce, G. Dugo, Pesticide and plasticizer residues in biological citrus essential oils from 2003-2004, Flavour Fragr. J. 21 (2006) 497-501. doi:10.1002/ffj.1663.

[16] M. Saitta, G. Di Bella, F. Salvo, S.L. Curto, G. Dugo, Organochlorine Pesticide Residues in Italian Citrus Essential Oils, 1991- 1996, J Agric Food Chem. 48 (2000) 797-801.

[17] M. Saitta, G. Di Bella, G. Dug, Pesticides and plasticizers in Citrus essential oils: An ordinary history of research, J. Essent. Oil Res. 24 (2012) 171-180. doi:10.1080/10412905.2012.659522.

[18] M.S.M. Garland, R.C. Menary, G.P.O. Box, Pesticide Minimum Residue Limits in Essential Oils - Report No 4, RIRDC, 2004.

[19] O. Tascone, C. Roy, J.-J. Filippi, U.J. Meierhenrich, Use, analysis, and regulation of pesticides in natural extracts, essential oils, concretes, and absolutes, Anal. Bioanal. Chem. (2013). doi:10.1007/s00216-013-7102-z. 
[20] R. Tisserand, T. Balacs, Essential oil safety: a guide for health care professionals, Churchill Livingstone, 1995.

[21] L. Alder, K. Greulich, G. Kempe, B. Vieth, Residue analysis of 500 high priority pesticides: Better by GC-MS or LC-MS/MS?, Mass Spectrom. Rev. 25 (2006) 838-865. doi:10.1002/mas.20091.

[22] M. Kuster, M.L. de Alda, D. Barceló, Analysis of pesticides in water by liquid chromatography-tandem mass spectrometric techniques, Mass Spectrom. Rev. 25 (2006) 900-916. doi:10.1002/mas.20093.

[23] Y. Picó, G. Font, M. José Ruiz, M. Fernández, Control of pesticide residues by liquid chromatography-mass spectrometry to ensure food safety, Mass Spectrom. Rev. 25 (2006) 917-960. doi:10.1002/mas.20096.

[24] K. Greulich, L. Alder, Fast multiresidue screening of 300 pesticides in water for human consumption by LC-MS/MS, Anal. Bioanal. Chem. 391 (2008) 183-197.

[25] Y. Fillâtre, D. Rondeau, A. Jadas-Hécart, P.Y. Communal, Advantages of the scheduled selected reaction monitoring algorithm in liquid chromatography/electrospray ionization tandem mass spectrometry multi-residue analysis of 242 pesticides: a comparative approach with classical selected reaction monitoring mode, Rapid Commun. Mass Spectrom. 24 (2010) 2453-2461. doi:10.1002/rcm.4649.

[26] S. Barrek, O. Paisse, M.F. Grenier-Loustalot, Analysis of pesticide residues in essential oils of citrus fruit by GC-MS and HPLC-MS after solid-phase extraction, Anal. Bioanal. Chem. 376 (2003) 157-161.

[27] H. Starr, U. Kiigemagi, L.C. Terriere, Insecticide Residues in Peppermint and Their Distillation with Peppermint Oil., J Agric Food Chem. 11 (1963) 482-486.

[28] R.D. Inman, U. Kiigemagi, M.L. Deinzer, Determination of carbofuran and 3hydroxycarbofuran residues in peppermint hay and peppermint oil, J Agric Food Chem. 31 (1983) 918-919.

[29] S.M. Garland, R.C. Menary, N.W. Davies, Dissipation of propiconazole and tebuconazole in peppermint crops (Mentha piperita (Labiatae)) and their residues in distilled oils, J Agric Food Chem. 47 (1999) 294-298.

[30] S. Chusaksri, S. Sutthivaiyakit, P. Sutthivaiyakit, Confirmatory determination of organochlorine pesticides in surface waters using LC/APCI/tandem mass spectrometry $\diamond$, Anal. Bioanal. Chem. 384 (2006) 1236-1245. doi:10.1007/s00216-005-0248-6.

[31] F. Hernandez, J.V. Sancho, O. Pozo, A. Lara, E. Pitarch, Rapid direct determination of pesticides and metabolites in environmental water samples at sub-[mu $\mathrm{g} / \mathrm{L}$ level by online solid-phase extraction-liquid chromatography-electrospray tandem mass spectrometry, J. Chromatogr. A. 939 (2001) 1-11.

[32] T. Chen, G. Chen, Identification and quantitation of pyrethroid pesticide residues in vegetables by solid-phase extraction and liquid chromatography/electrospray ionization ion trap mass spectrometry, Rapid Commun. Mass Spectrom. 21 (2007) 1848-1854. doi: $10.1002 / \mathrm{rcm} .3027$.

[33] R.M. González-Rodriguez, R. Rial-Otero, B. Cancho-Grande, J. Simal-Gándara, Determination of 23 pesticide residues in leafy vegetables using gas chromatographyion trap mass spectrometry and analyte protectants, J. Chromatogr. A. 1196 (2008) 100109.

[34] E. Björklund, S. Sporring, K. Wiberg, P. Haglund, C. Holst, New strategies for extraction and clean-up of persistent organic pollutants from food and feed samples using selective pressurized liquid extraction, TrAC Trends Anal. Chem. 25 (2006) 318325.

[35] A. Garrido Frenich, I. Martínez Salvador, J.L. Martínez Vidal, T. López-López, Determination of multiclass pesticides in food commodities by pressurized liquid 
extraction using GC-MS/MS and LC-MS/MS, Anal. Bioanal. Chem. 383 (2005) 11061118. doi:10.1007/s00216-005-0139-x.

[36] C. Blasco, G. Font, Y. Picó, Analysis of pesticides in fruits by pressurized liquid extraction and liquid chromatography-ion trap-triple stage mass spectrometry, J. Chromatogr. A. 1098 (2005) 37-43.

[37] P. Payá, M. Anastassiades, D. Mack, I. Sigalova, B. Tasdelen, J. Oliva, et al., Analysis of pesticide residues using the Quick Easy Cheap Effective Rugged and Safe (QuEChERS) pesticide multiresidue method in combination with gas and liquid chromatography and tandem mass spectrometric detection, Anal. Bioanal. Chem. 389 (2007) 1697-1714.

[38] M. Kirchner, R. Húšková, E. Matisová, J. Mocák, Fast gas chromatography for pesticide residues analysis using analyte protectants, J. Chromatogr. A. 1186 (2008) 271-280.

[39] NF EN 15662, Méthode polyvalente de détermination des résidus de pesticides par CG$\mathrm{SM}$ et SL/SM/SM avec extraction/partition avec de l'acétonitrile et nettoyage par SPE dispersés: méthode QuEChERS, AFNOR, Paris (France), 2009.

[40] R. Romero-González, A.G. Frenich, J.L.M. Vidal, Multiresidue method for fast determination of pesticides in fruit juices by ultra performance liquid chromatography coupled to tandem mass spectrometry, Talanta. 76 (2008) 211-225. doi:10.1016/j.talanta.2008.02.041.

[41] M.D. Hernando, C. Ferrer, M. Ulaszewska, J.F. García-Reyes, A. Molina-Díaz, A.R. Fernández-Alba, Application of high-performance liquid chromatography-tandem mass spectrometry with a quadrupole/linear ion trap instrument for the analysis of pesticide residues in olive oil, Anal. Bioanal. Chem. 389 (2007) 1815-1831. doi:10.1007/s00216007-1464-z.

[42] B. Kmellár, P. Fodor, L. Pareja, C. Ferrer, M.A. Martínez-Uroz, A. Valverde, et al., Validation and uncertainty study of a comprehensive list of 160 pesticide residues in multi-class vegetables by liquid chromatography-tandem mass spectrometry, J. Chromatogr. A. 1215 (2008) 37-50. doi:10.1016/j.chroma.2008.10.121.

[43] Y. Fillâtre, D. Rondeau, B. Bonnet, A. Daguin, A. Jadas-Hécart, P.-Y. Communal, Multiresidue Analysis of Multiclass Pesticides in Lavandin Essential Oil by LC/MS/MS Using the Scheduled Selected Reaction Monitoring Mode, Anal. Chem. 83 (2011) 109117. doi:10.1021/ac1018292.

[44] Y. Fillâtre, D. Rondeau, A. Daguin, A. Jadas-Hecart, P.-Y. Communal, Multiresidue determination of 256 pesticides in lavandin essential oil by LC/ESI/sSRM: advantages and drawbacks of a sampling method involving evaporation under nitrogen, Anal. Bioanal. Chem. 406 (2014) 1541-1550. doi:10.1007/s00216-013-7553-2.

[45] A. Kaufmann, P. Butcher, K. Maden, M. Widmer, K. Giles, D. Uría, Are liquid chromatography/electrospray tandem quadrupole fragmentation ratios unequivocal confirmation criteria?, Rapid Commun. Mass Spectrom. 23 (2009) 985-998. doi:10.1002/rcm.3959.

[46] F. Hernandez, J.V. Sancho, O.J. Pozo, Direct determination of alkyl phosphates in human urine by liquid chromatography/electrospray tandem mass spectrometry, Rapid Commun. Mass Spectrom. 16 (2002) 1766-1773. doi:10.1002/rcm.790.

[47] J.W. Hager, others, A new linear ion trap mass spectrometer, Rapid Commun. Mass Spectrom. 16 (2002) 512-526.

[48] C.A. Mueller, W. Weinmann, S. Dresen, A. Schreiber, M. Gergov, Development of a multi-target screening analysis for 301 drugs using a QTrap liquid chromatography/tandem mass spectrometry system and automated library searching, Rapid Commun. Mass Spectrom. 19 (2005) 1332-1338. doi:10.1002/rcm.1934. 
[49] B. Kmellar, L. Abranko, P. Fodor, S.J. Lehotay, Routine approach to qualitatively screening 300 pesticides and quantification of those frequently detected in fruit and vegetables using liquid chromatography tandem mass spectrometry (LC-MS/MS), Food Addit. Contam. Part A. 27 (2010) 1415-1430. doi:10.1080/19440049.2010.490791.

[50] AFNOR NF ISO 855, Huile essentielle de citron [Citrus limon (L.) Burm. f.], AFNOR, Paris (France), 2004.

[51] AFNOR NF T 75-254, Huile essentielle de cyprès (Cupressus sempervirens Linnaeus), AFNOR, Paris (France), 1992.

[52] AFNOR NF T 75-305, Huile essentielle de lavandin super [Lavandula angustifolia P.Miller x Lavandula latifolia (Linnaeus fils) Medikus], AFNOR, Paris (France), 1992.

[53] S. Walorczyk, B. Gnusowski, Development and validation of a multi-residue method for the determination of pesticides in honeybees using acetonitrile-based extraction and gas chromatography-tandem quadrupole mass spectrometry, J. Chromatogr. A. 1216 (2009) 6522-6531. doi:10.1016/j.chroma.2009.07.045.

[54] C. Lentza-Rizos, E.J. Avramides, F. Cherasco, Low-temperature clean-up method for the determination of organophosphorus insecticides in olive oil, J. Chromatogr. A. 912 (2001) 135-142.

[55] S.M. Goulart, M.E.L.R. de Queiroz, A.A. Neves, J.H. de Queiroz, Low-temperature clean-up method for the determination of pyrethroids in milk using gas chromatography with electron capture detection, Talanta. 75 (2008) 1320-1323. doi:10.1016/j.talanta.2008.01.058.

[56] S. Chen, X. Yu, X. He, D. Xie, Y. Fan, J. Peng, Simplified pesticide multiresidues analysis in fish by low-temperature cleanup and solid-phase extraction coupled with gas chromatography/mass spectrometry, Food Chem. 113 (2009) 1297-1300. doi:10.1016/j.foodchem.2008.08.045.

[57] European Commission Health and Consumer Protection, HEALTH \& CONSUMER PROTECTION DIRECTORATE, SANCO/ 12571 /2013 Guidance document on analytical quality control and validation procedures for pesticide residues analysis in food and feed, (2013).

[58] US Environmental Protection Agency Office of Pesticide Programs, Index to Pesticide Chemical Names, Part 180 Tolerance Information, and Food and Feed Commodities (by Chemical Name), (2010).

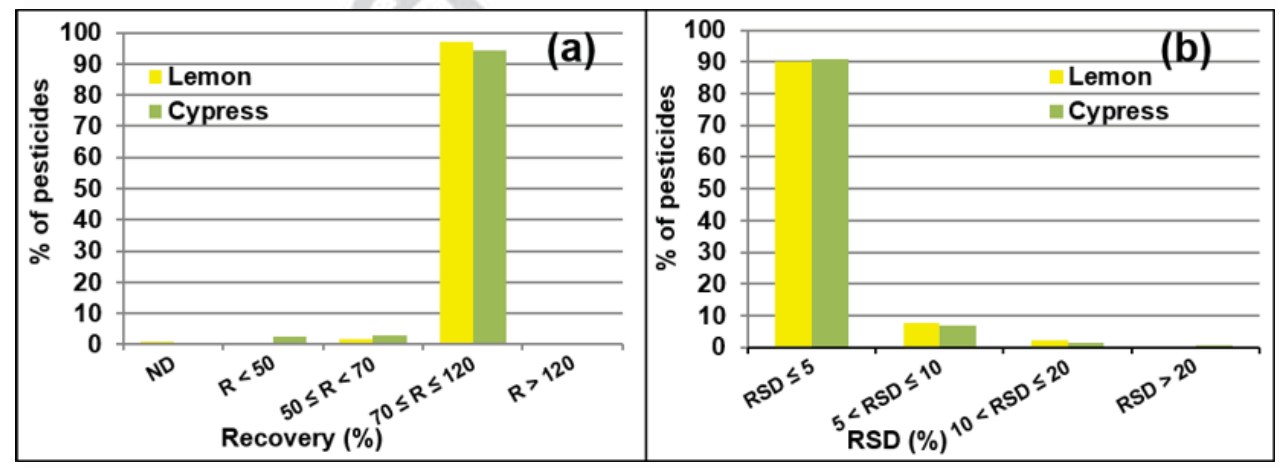



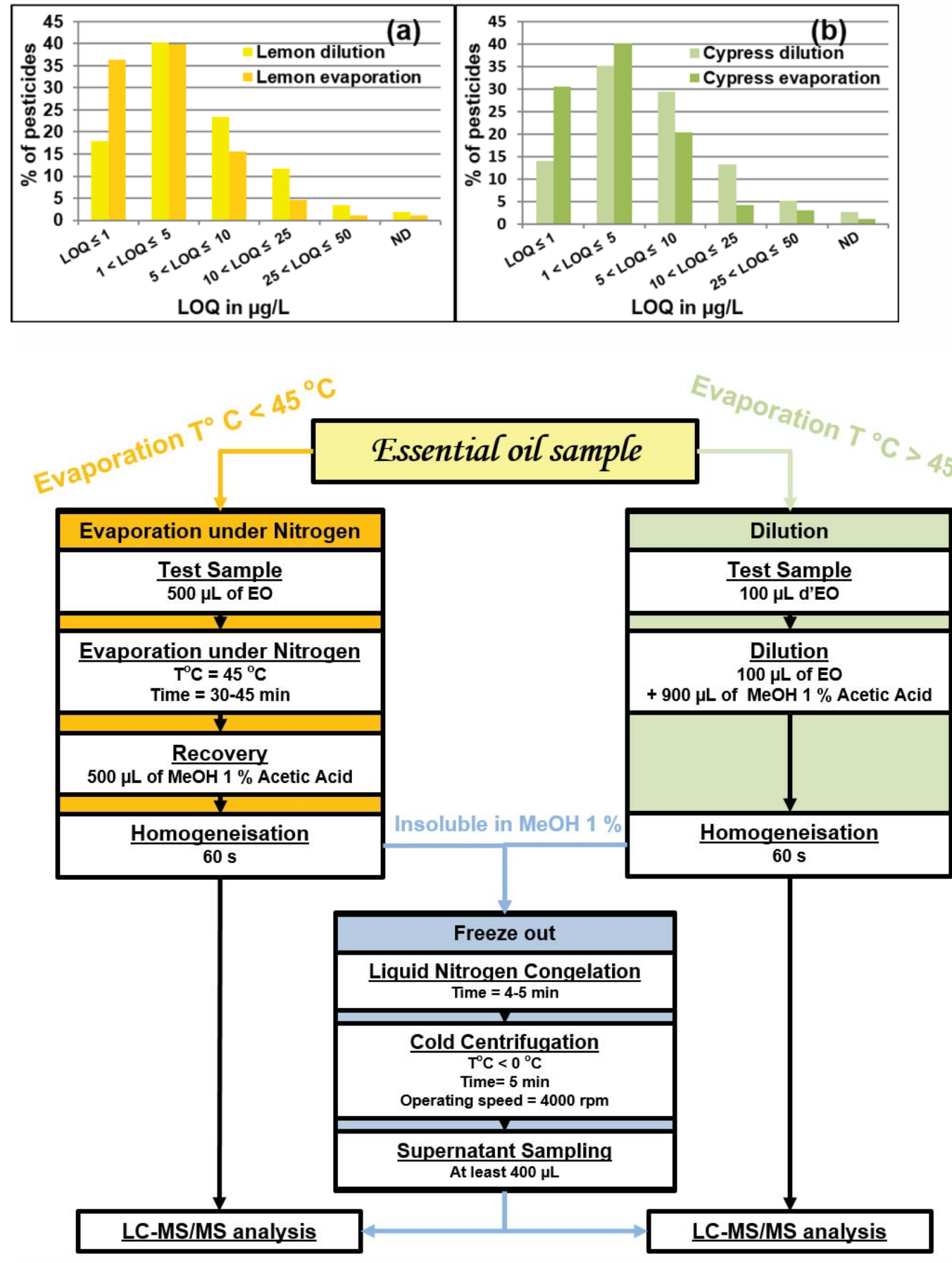


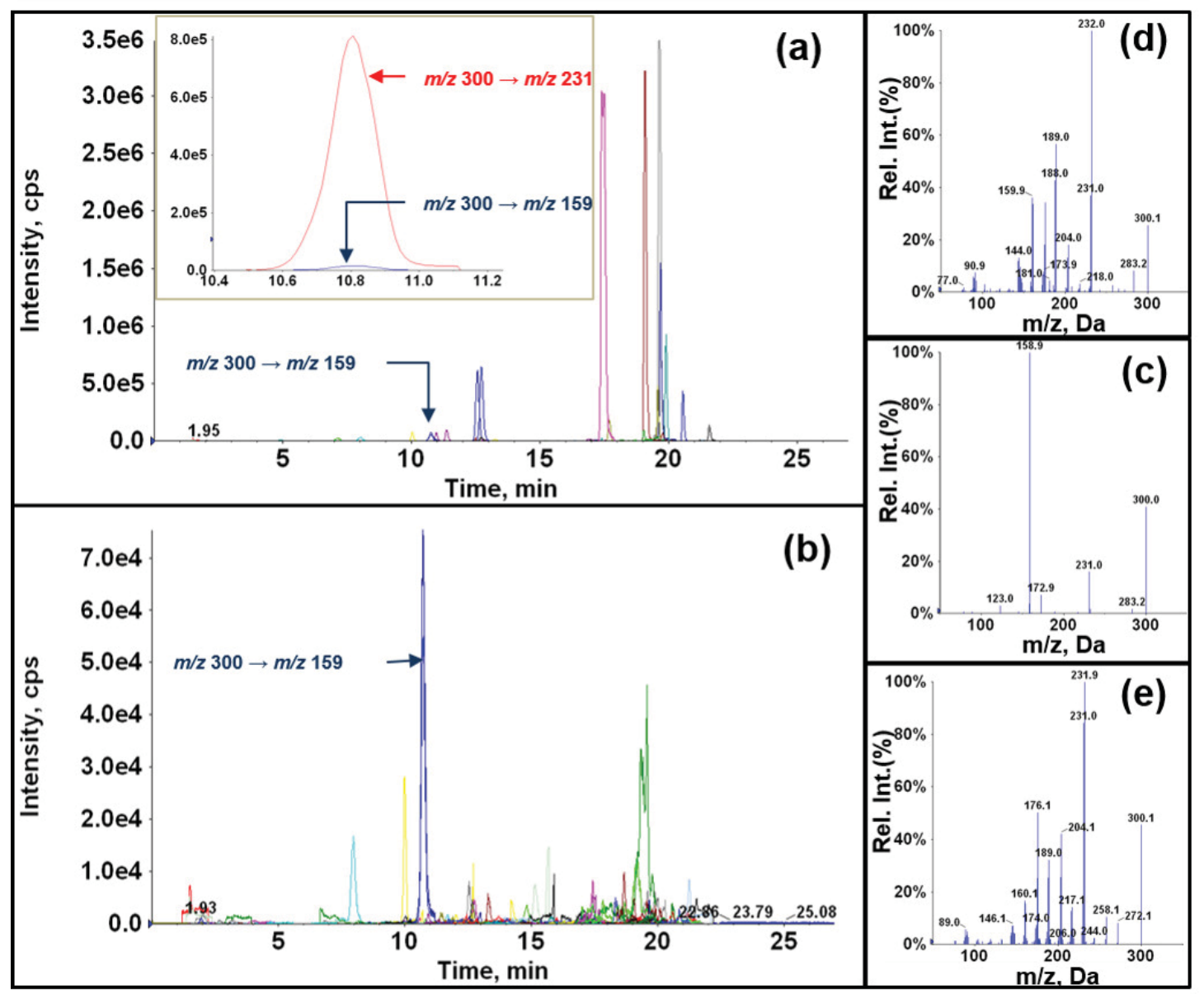

Highlights

A guideline for quantifying pesticides in various essential oils by LC-MS/MS is proposed.

Sample preparation by evaporation under nitrogen gives better LOQs for essential oils with low evaporation temperature.

Use of Enhanced Product Ion scan for positive confirmation is discussed.

Application to the analyses of two lemon essential oils allowed detecting 22 pesticides.

The concentration of pesticides in lemon essential oils can reach several mg/L. 


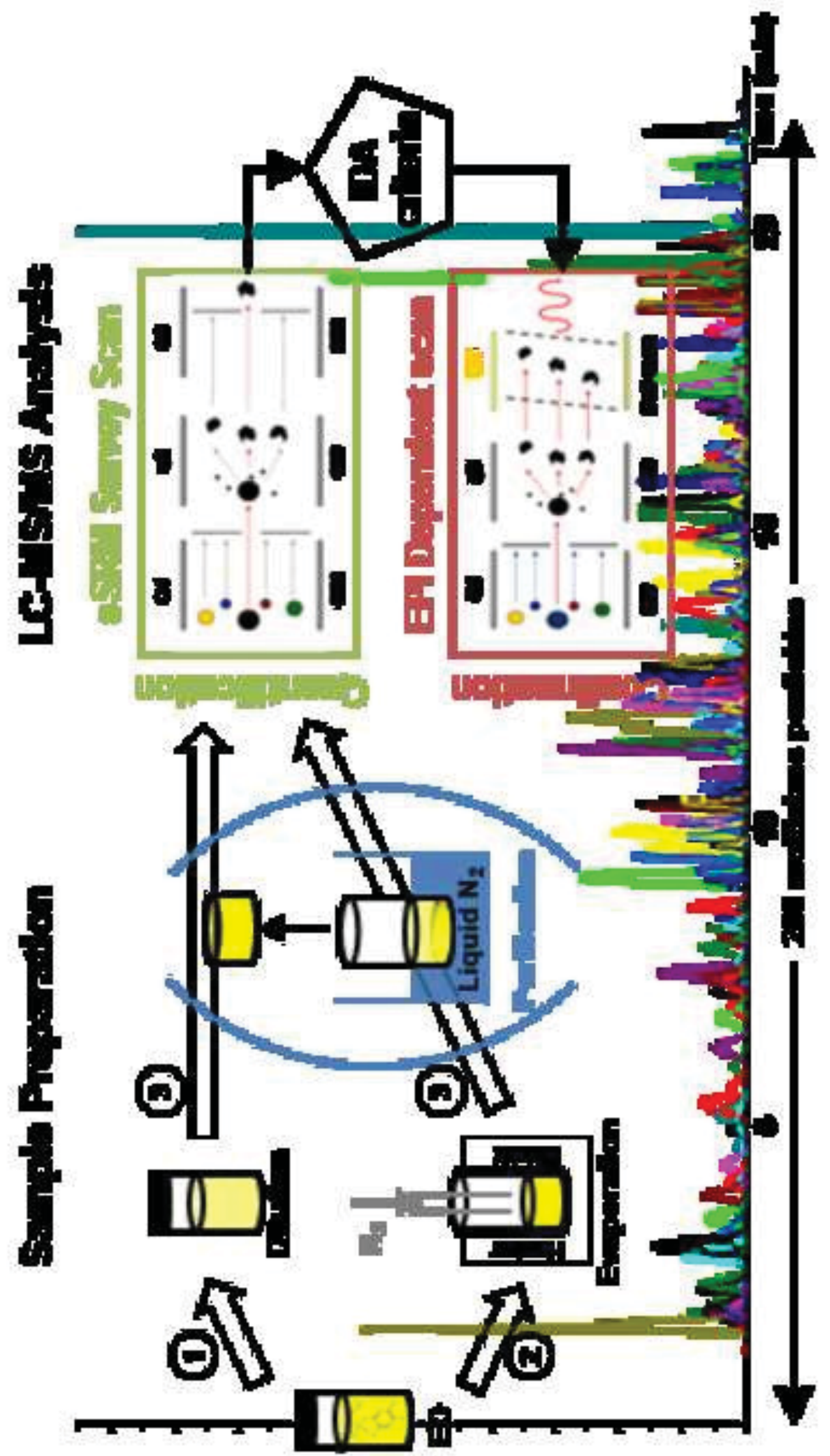

